\title{
INFLUENNCIA DO PACOTE DE PROPRIEDADES TERMOFÍSICAS NO PROJETO DE UMA UNIDADE DE SEPARAÇÃO BUTANOL-ÁGUA
}

\author{
R. M. NICODEMOS ${ }^{1}$ e A. J. ASSIS ${ }^{1}$ \\ ${ }^{1}$ Universidade Federal de Uberlândia - Faculdade de Engenharia Química - Núcleo de \\ Modelagem, Controle e Otimização de Processos \\ E-mail: renata.mnicodemos@gmail.com
}

\begin{abstract}
RESUMO - A simulação de plantas inteiras tem se tornado cada vez mais importante na análise de processos. Embora a escolha do pacote de propriedades termofísicas seja uma etapa crucial na simulação, nem sempre dá-se a devida importância a tal escolha. Neste trabalho, analisa-se a influência da escolha de diferentes pacotes de propriedades termodinâmicas usados para descrever os equilíbrios líquido-vapor (ELV) e líquido-líquido (ELL), sobre o desempenho da unidade. Analisa-se também como o ELV e o ELL afetam o cálculo da volatilidade relativa, a taxa de transferência de massa e o gasto energético do processo, sendo este último uma medida indireta do custo operacional da planta. As simulações foram efetuadas nos simuladores de processos $\mathrm{COCO}$ e ChemSep. Os valores obtidos foram validados com dados experimentais da literatura e mostram que o modelo que melhor descreve o ELV é o NRTL e para o ELL é UNIFAC. Assim, mostra-se como a termodinâmica impacta parâmetros operacionais importantes em processos simulados.
\end{abstract}

\section{INTRODUÇÃO}

Os simuladores de processos podem ser utilizados como poderosa ferramenta de análise, síntese, simulação, otimização,etc, tanto no setor industrial, quanto no meio acadêmico. O que permitiu a abrangência do uso dos simuladores de processos em tantas áreas foram os bancos de propriedades termofísicas, cada vez mais precisos e a facilidade de uso, já que contem as principais operações unitárias que permitem reproduzir com alto grau de fidelidade os processos convencionais.

Apesar das funcionalidades dos simuladores de processos, existem restrições que limitam sua utilização de forma mais ampla. Para a simulação de uma planta química, é necessário, além das condições operacionais, um banco de dados com as propriedades termofísicas das substâncias envolvidas no processo. Geralmente, tais bancos de dados são constituídos de equações empíricas, equações de estado, modelos de energia, etc. Caso o cálculo das propriedades não seja acurado, o resultado obtido por meio da simulação não será exato e automaticamente a análise e a otimização também serão prejudicadas

Alguns trabalhos na literatura abordam a escolha do pacote termodinâmico em simuladores de processos. Por exemplo, CARLSON (1996) lista tipos de modelos termodinâmicos, como equações de estado e modelos de cálculo do coeficiente de atividade, de acordo com as suas aplicações para cada tipo de sistema. Já HILL e JUSTICE (2011), abordam a necessidade da validação dos dados obtidos na simulação. Nos trabalhos de PINTO (1998) e PINTO (2001) é apresentada uma abordagem teórica em que é possível calcular o custo das variações dos parâmetros do processo sobre a economia deste. Essa 
abordagem pode tanto ser utilizada para otimização, quanto em escolhas do planejamento experimental.

O objetivo deste trabalho é analisar a influência do pacote de propriedades termofísicas no projeto de uma unidade de separação butanol-água. Para tal, foi investigado como a volatilidade relativa e a taxa de transferência de massa são influenciadas pela escolha do modelo termodinâmico utilizado para descrever o decantador. Também foi verificada a influência sobre o gasto energético estimado do processo, como uma medida do custo operacional do mesmo, e o efeito da escolha do pacote termodinâmico para os equilíbrios líquido-vapor e líquido-líquido.

\section{METODOLOGIA}

\subsection{SIMULADOR DE PROCESSOS}

As simulações realizadas neste trabalho foram feitas com o simulador de processos COCO (Cape Open to Cape Open), que é um simulador gratuito de processos no estado estacionário. O simulador COCO é dividido em quatro sub-aplicativos:

- COFE - é a interface gráfica para o usuário; possuiu um algoritmo de solução sequencial e exibe os dados das correntes e dos equipamentos.

- TEA - é o pacote de dados termodinâmicos baseado na biblioteca do ChemSep incluindo aproximadamente 430 substâncias e mais de 100 métodos de cálculo de propriedades.

- COUSCOUS - é o pacote que engloba as operações unitárias disponíveis no simulador.

- $\mathrm{CORN}$ - pacote de reações químicas; a partir deste pacote é possível especificar o tipo de cinética ou uma reação de equilíbrio.

\subsection{ESTUDO DE CASO: SEPARAÇÃO DE BUTANOL-ÁGUA}

O butanol e a água formam um azeótropo heterogêneo líquido-líquido-vapor de mínimo e pode ser separado usando duas colunas de destilação simples, mais um decantador. $\mathrm{Na}$ primeira coluna destila-se a mistura até próximo ao ponto de azeótropo e através do equilíbrio líquido-líquido que se forma no decantador, com duas fases distintas, uma aquosa e outra orgânica, é possível terminar a separação em uma segunda coluna de destilação, alimentando a fase orgânica nesta. Isto permite "quebrar" o azeótropo, nesta mistura. A Figura 1 mostra o fluxograma da planta de separação de butanol-água. A simulação é uma adaptação de Luyben et al (2008).

A corrente de alimentação, "Feed", com vazão de $1000 \mathrm{kmol} / \mathrm{h}$, sendo $98 \%$ de água e $2 \%$ de butanol, a 0,5 atm e $78,85^{\circ} \mathrm{C}$ passa por uma coluna de destilação simples, "C1", com 10 estágios de equilíbrio, que separa duas correntes: "Water", com 99\%, em base molar, de água na fase líquida e a corrente de topo com $15 \%$, em base molar, de butanol na fase vapor, ambas as correntes a $0,5 \mathrm{~atm}$. A corrente de topo da coluna " $\mathrm{C} 1$ " é somada à corrente de topo da coluna "C2" por um "Mixer" e enviada para o decantador "Decanter".É separada na corrente "Organic" (rica em butanol), corrente de alimentação da coluna "C2", e "Aqueous" (corrente rica em água), corrente de entrada no topo da coluna " $\mathrm{C} 1$ ". Ambas as correntes Organic e Aqueous, estão a 1atm. Por fim, tem-se a corrente "Butanol", corrente de fundo da coluna 
"C2", com butanol a 99\% em base molar. Neste processo, a operação unitária chave é o decantador, pois é nele que ocorre a quebra do azeótropo.

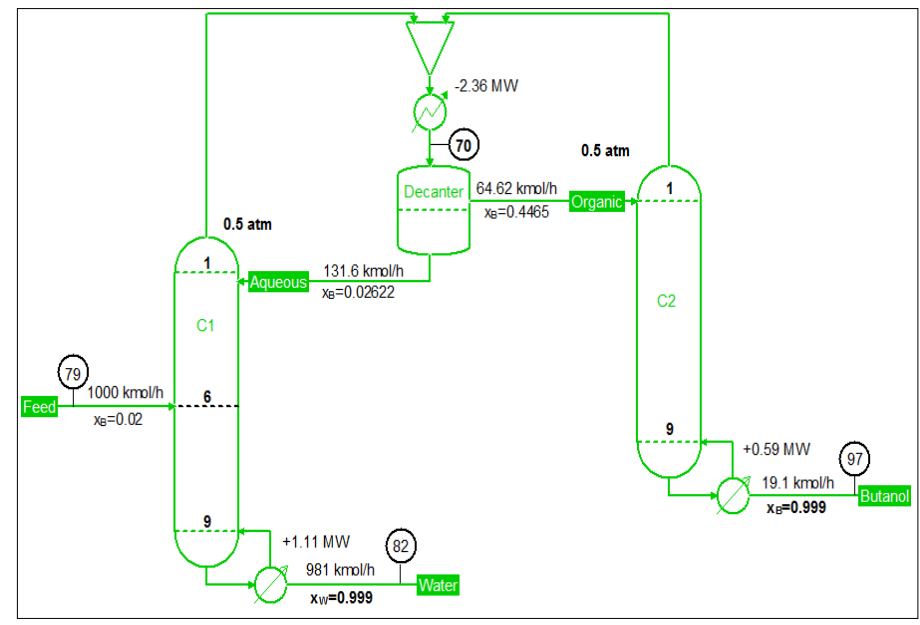

Figura 1. Fluxograma da planta industrial de separação de butanol e água

Os dados obtidos de equilíbrio líquido-vapor usados nas simulações com modelos diferentes foram comparados com os dados disponíveis na literatura. Os dados experimentais encontrados estavam a pressão de 1,0 atm enquanto as simulações ocorrem em a 0,5 e 1,0 atm. Como os modelos analisados não apresentam pressão nas suas equações, a influência desta variável sobre o cálculo dos coeficientes de atividade é pequena e deste modo tais modelos foram validados a uma pressão diferente das de simulação. Ademais, as pressões usadas são baixas (próximas a 1atm). Para a análise dos dados de equilíbrio líquido-vapor, os modelos alterados foram o da simulação como um todo. Já para a análise a influência dos modelos termofísicos sobre o processo e equilíbrio líquido-líquido, a modificação foi apenas no modelo termodinâmico do decantador.

As especificações dos produtos e de projeto nas colunas de destilação permaneceram inalteradas. Portanto, para analisar a influência dos modelos termofísicos sobre o processo foram escolhidas duas propriedades que medem o grau de facilidade ou dificuldade da separação dentro das colunas de destilação, a saber a volatilidade relativa e a taxa de transferência de massa. E, a fim de medir indiretamente a influência dos modelos termofísicos sobre o custo operacional do processo, foram consideradas as cargas térmicas dos trocadores de calor presentes, pois como as condições de projeto ficaram inalteradas, a fim de se obter as mesmas especificações para os produtos, as cargas térmicas devem se reposicionar para novos patamares.

\section{RESULTADOS}

Para modelar a fase vapor, usou a equação dos gases ideais $(\mathrm{P}=0,5 \mathrm{~atm})$, com a qual se calculou a densidade, a entalpia, a entalpia de formação, a entropia, a entropia de formação, a fugacidade, o coeficiente de fugacidade e o volume, propriedades usadas pelo simulador. Para modelar a fase líquida, usou-se um modelo de energia livre de Gibbs de excesso, com o qual se calculou a atividade, o coeficiente de atividade, a entropia, a entropia de formação e a entalpia de excesso. Para o decantador, o modelo utilizado para o cálculo do coeficiente de atividade é o UNIFAC com a abordagem $\gamma-\gamma$ para o cálculo do valor de K.

A Figura 2 mostra os resultados obtidos para o ELV com os modelos UNIFAC, Wilson, NRTL e UNIQUAC para o cálculo da fase líquida e a lei dos gases ideais para a fase vapor comparados com os dados experimentais de DDBST GmbH (2013). Os quatro modelos 
analisados conseguiram descrever a existência do azeótropo, mas para o modelo de Wilson, o ponto de azeótropo está a uma temperatura mais alta do que os dados experimentais. Apenas observando os dados no gráfico não é possível determinar qual modelo se aproxima mais dos dados experimentais. Portanto, foi calculado o desvio padrão médio entre os dados experimentais e os resultados obtidos pelos modelos. Os valores estão disponíveis na Tabela 1.

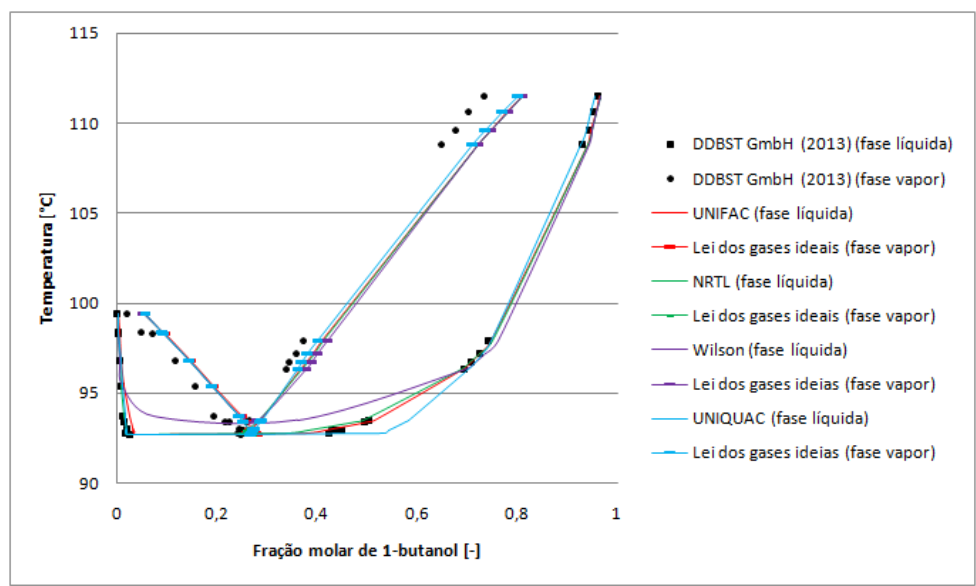

Figura 2- Diagramas ELV a pressão constante comparando dados experimentais e modelos para a mistura binária butanol-água na fase líquida

Tabela 1 - Erro relativo médio entre dados experimentais e valores obtidos pelos modelos para a fase líquida

\begin{tabular}{|c|c|c|}
\hline Modelo & $\mathrm{n}$ & Erro relativo médio [\%] \\
\hline UNIFAC & 25 & 41,80 \\
\hline Wilson & 15 & 44,32 \\
\hline NRTL & 25 & 13,02 \\
\hline UNIQUAC & 25 & 22,90 \\
\hline
\end{tabular}

O menor erro relativo médio calculado foi para o modelo NRTL, 13,02\%. As equações UNIFAC e UNIQUAC são adequadas para prever atividade de sistemas binários água/orgânicos (gás ideal/líquido polar). Já para prever a interação binária, as equações NRTL e Wilson são melhores. O que está sendo avaliado é a interação da mistura binária água/butanol no equilíbrio líquido-vapor, já que se trata de uma separação, portanto, baseando-se a teoria, NRTL é o melhor modelo para este sistema.

Para a fase vapor, como as pressões de operação são baixas (0,5 e 1,0 atm), os resultados gráficos são muito semelhantes. Portanto, para esta fase os resultados serão apresentados apenas pelo desvio padrão médio.

A Tabela 2 mostra os erros relativos médios obtidos para cada equação de estado utilizada para fase vapor. O menor erro encontrado foi para a Lei dos Gases Ideais com o valor de $23,51 \%$, a equação utilizada no default da simulação. Os valores obtidos para os erros relativos médios são bastante semelhantes. Este comportamento pode ser explicado pelo fato da simulação acontecer a baixas pressões, os únicos pontos em que a simulação está a 1atm é nas colunas de destilação, $\mathrm{C} 1$ e C2.

Os dados de equilíbrio líquido-líquido obtidos na simulação foram comparados com os dados experimentais encontrados na literatura (GÓRAL et al,. 2006) como é possível ver na Figura 3. O intervalo analisado é de 50 a $100^{\circ} \mathrm{C}$, já que o decantador opera a aproximadamente $70^{\circ} \mathrm{C}$. Os modelos usados nesta etapa foram UNIFAC, UNIQUAC e 
NRTL. Os modelos Margules e Wilson não geraram duas fases líquidas heterogêneas, impossibilitando o processo de separação.

Tabela 2 - Erro relativo médio entre dados experimentais e valores obtidos pelos modelos para a fase vapor

\begin{tabular}{|c|c|c|}
\hline Modelo & $\mathrm{n}$ & Erro relativo médio [\%] \\
\hline Lei dos Gases Ideias & 25 & 23,51 \\
\hline Peng Robinson & 25 & 24,06 \\
\hline RedlichKwong & 25 & 24,11 \\
\hline HaydenO'Connell & 25 & 24,19 \\
\hline
\end{tabular}

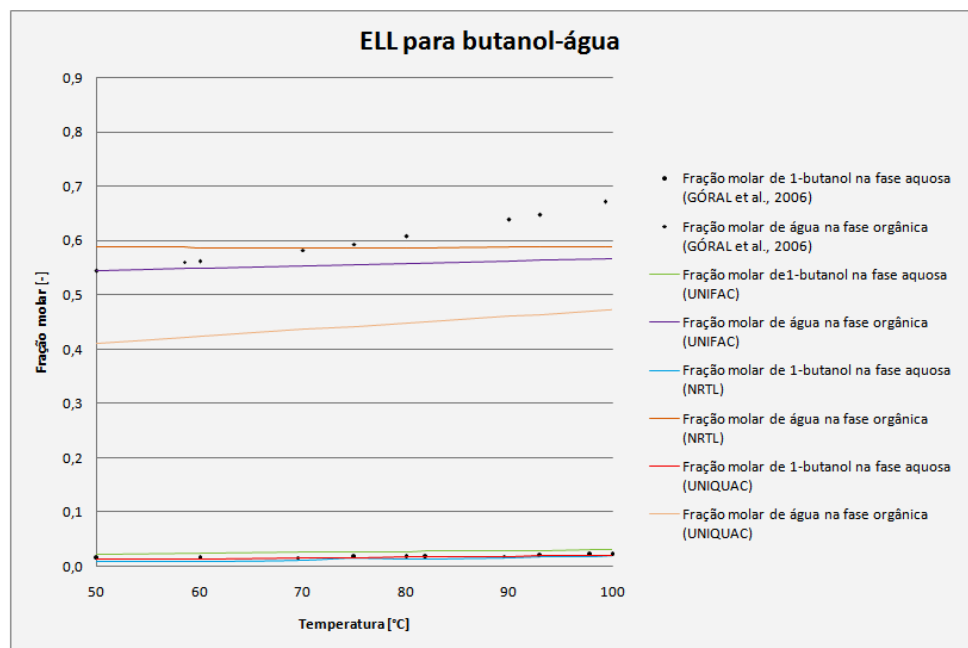

Figura 3- Diagramas ELL a pressão constante comparando dados experimentais e modelos para a mistura binária butanol-água

As curvas superiores representam a fração molar de água na fase orgânica, rica em butanol, em relação à temperatura. Os dados calculados não se aproximaram satisfatoriamente dos dados experimentais. Já as curvas que indicam a fase aquosa, curvas próximas à abscissa, o comportamento dos modelos foi próximo aos dados da literatura.

A Tabela 3 mostra os valores dos erros relativos médios encontrados para os modelos analisados. Observando os resultados é possível afirmar que o menor erro relativo encontrado é para o modelo UNIFAC para ambas as fases.

Tabela 3- Erro relativo médio entre dados experimentais e valores obtidos pelos modelos para o ELL

\begin{tabular}{|c|c|c|c|}
\hline Modelo & Fase & $\mathrm{n}$ & Erro relativo médio [\%] \\
\hline \multirow{2}{*}{ UNIFAC } & orgânica & 30 & 25,87 \\
\cline { 2 - 4 } & aquosa & 30 & 7,55 \\
\hline \multirow{2}{*}{ UNIQUAC } & orgânica & 30 & 69,50 \\
\cline { 2 - 4 } & aquosa & 30 & 12,44 \\
\hline \multirow{2}{*}{ NRTL } & orgânica & 30 & 32,40 \\
\cline { 2 - 4 } & aquosa & 30 & 20,39 \\
\hline
\end{tabular}

Para analisar o efeito do pacote termodinâmico sobre o projeto do processo foi alterada a forma de cálculo do coeficiente de atividade do decantador, operação-chave na separação butanol-água simulada. Os modelos utilizados foram: NRTL, UNIQUAC, UNIFAC, 
UNIFAC modificado e Margules. A Tabela 4 mostra os valores calculados com cada modelo para as correntes "Organic" e "Aqueous" para as substâncias envolvidas no processo, butanol e água.

Tabela 4 - Valores calculados de coeficiente de atividade para cada modelo termodinâmico analisado somente para o decantador

\begin{tabular}{|c|c|c|c|c|c|c|}
\hline \multirow{2}{*}{ Corrente } & \multirow{2}{*}{ Substância } & \multicolumn{5}{|c|}{ Coeficiente de Atividade (fase líquida) } \\
\cline { 3 - 7 } & & NRTL & UNIQUAC & UNIFAC & $\begin{array}{c}\text { UNIFAC } \\
\text { modificado }\end{array}$ & Margules \\
\hline \multirow{2}{*}{ Aqueous } & Butanol & 24,8837 & 23,6015 & 23,4958 & 22,5997 & 24,4545 \\
\cline { 2 - 7 } & Água & 1,0066 & 1,0079 & 1,0081 & 1,0092 & 1,0070 \\
\hline Organic & Butanol & 1,1280 & 1,1663 & 1,3879 & 1,1741 & 1,0466 \\
\cline { 2 - 7 } & Água & 2,2658 & 2,1491 & 1,7869 & 1,0092 & 2,7061 \\
\hline
\end{tabular}

As correntes "Organic" e "Aqueous" são as de saída do decantador e, posteriormente, serão as correntes de entrada das colunas de destilação $\mathrm{C} 2$ e $\mathrm{C} 1$, respectivamente, o que justifica uma análise mais detalhada de algumas propriedades das colunas de destilação. Sabendo-se que as equações UNIFAC e UNIQUAC são mais adequadas para prever atividade de sistemas binários água/orgânicos (gás ideal/líquido polar), pode-se dizer que, para este quesito, um dos dois modelos seria o mais indicado.

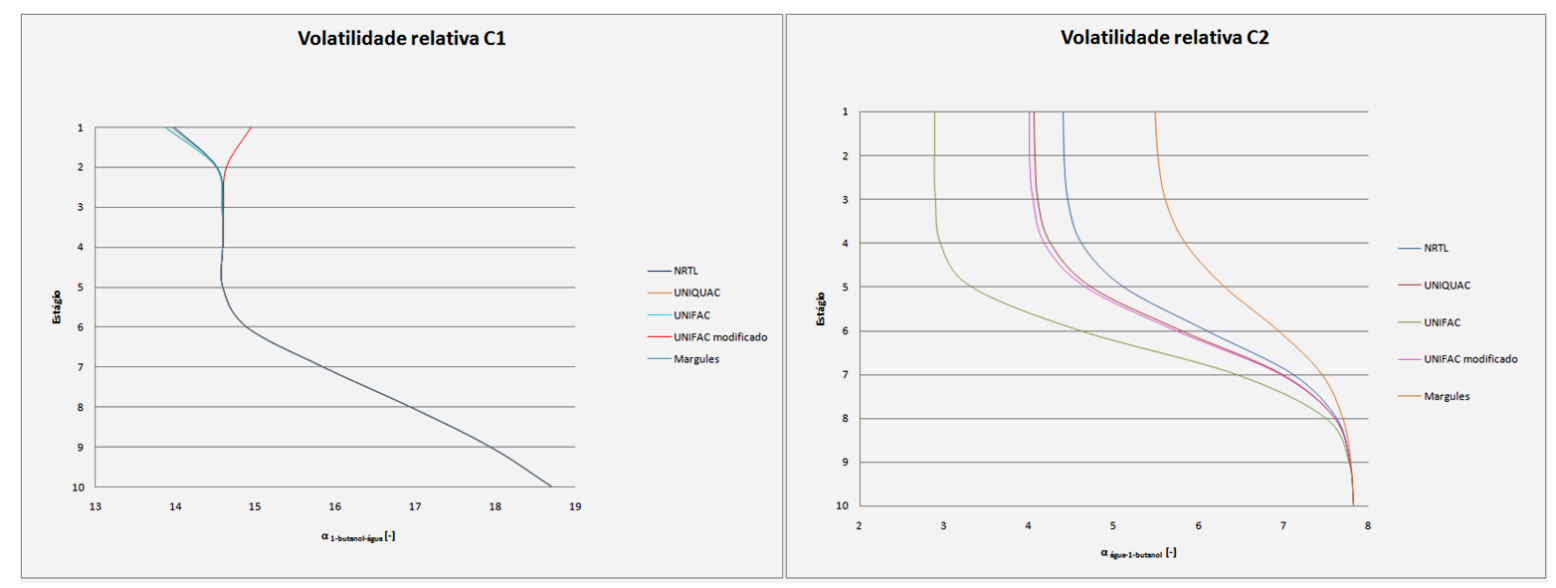

Figura 4- Volatilidade relativa butanol-água para as colunas de destilação C1 e C2 para os modelos termodinâmicos analisados

A Figura 4 mostra as volatilidades relativas butanol-água encontradas pelos modelos de cálculo de coeficiente de atividade. Os modelos utilizados foram NRTL, UNIQUAC, UNIFAC, UNIFAC modificado e Margules. Quanto maior a volatilidade relativa mais favorável será a separação. A primeira coluna, C1, apresenta maiores valores de volatilidade relativa em relação à segunda coluna de destilação, C2. Para a primeira coluna, os modelos apresentam resultados bastante semelhantes para a volatilidade relativa, exceto para os dois estágios iniciais, o que mostra que a escolha do modelo termodinâmico do decantador terá pouca influência sobre a composição calculada na corrente de fundo da coluna C1. Já para a segunda coluna, C2, os valores de volatilidade relativa coincidem apenas no estágio 10 e são valores completamente diferentes nos outros estágios. Estes resultados indicam uma região grande de incerteza no projeto da coluna C2, já que para cada modelo é apresentado um resultado diferente. Como esta variação ocorre nos estágios superiores da coluna, isto interfere diretamente na eficiência do decantador que receberá a corrente de topo da coluna como corrente de entrada. 
A Figura 5 mostra as taxas de transferência de massa dentro das colunas $\mathrm{C} 1$ e C2, respectivamente, para os modelos NRTL, UNIQUAC, UNIFAC, UNIFAC modificado e Margules para o cálculo do coeficiente de atividade no decantador. Assim como para a volatilidade relativa da coluna de destilação $\mathrm{C} 1$, a taxa de transferência de massa para a coluna $\mathrm{C} 1$ é igual para os estágios inferiores da coluna e se altera a partir do segundo estágio como é possível observar. O modelo UNIFAC modificado é o que mais apresenta desvios em relação aos demais modelos ocorrendo variações também entre os estágios 4 e 5 e a partir do estágio 3. Já para coluna C2 nota-se que os valores calculados para a taxa de transferência de massa em $\mathrm{C} 2$, para cada modelo analisado, foram diferentes. O único ponto que os valores coincidem é no estágio 2. O modelo que mais desvia em relação aos outros é o UNIFAC, modelo original da simulação. Neste caso, a região de fundo da coluna C2 é a região de maior incerteza do projeto. UNIFAC apresenta as maiores taxas de transferência.

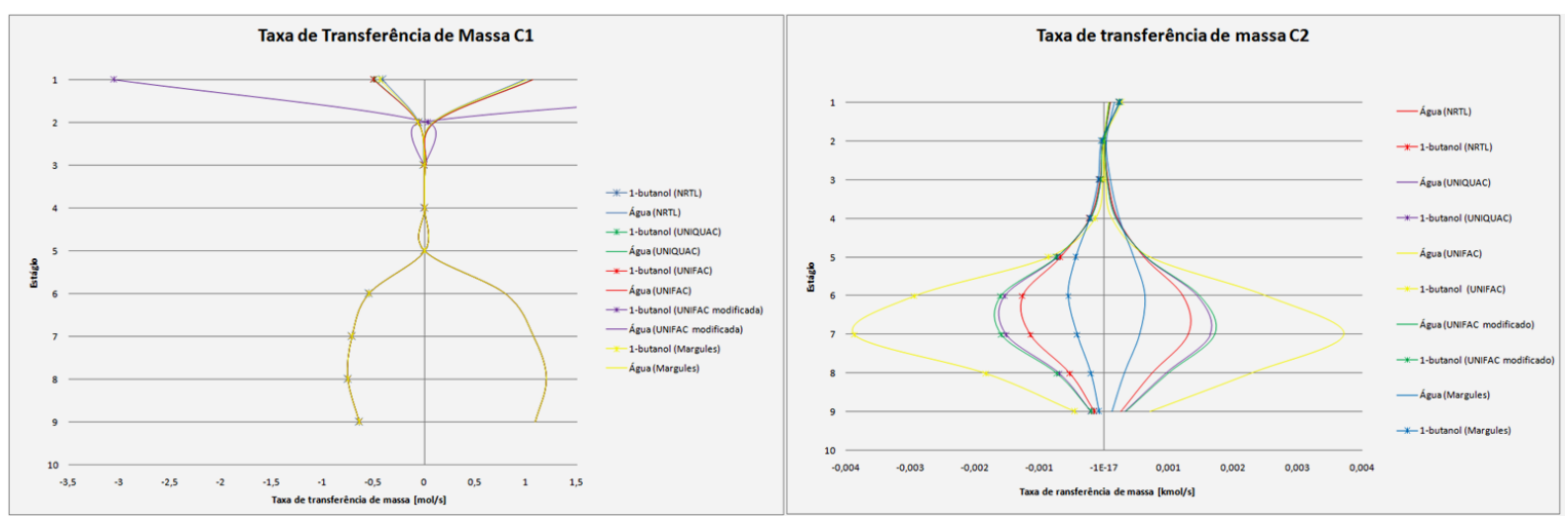

Figura 5- Taxa de transferência de massa para butanol-água para a coluna $\mathrm{C} 1$ de acordo com os modelos termodinâmicos analisados

Por último, foram calculados e analisados os valores das cargas térmicas das colunas de destilação e do trocador de calor que está antes do decantador, como uma medida indireta do custo operacional do processo. A carga térmica do trocador de calor da coluna de destilação C1 não se mostrou sensível à alteração do modelo termodinâmico do decantador, já que para tal coluna os modelos são coincidentes no fundo desta, conforme mostrado na Figura 4. Já para os dois outros trocadores de calor, os valores das cargas térmicas variam sensivelmente, como é mostrado na Tabela 5.

Pelos resultados apresentados, percebe-se a sensibilidade dos resultados de gasto energético obtidos na simulação ao modelo termodinâmico do decantador, fixados o número de estágios teóricos das colunas e as composições dos produtos. Apenas para o trocador de calor da coluna de destilação $\mathrm{C} 2$, a diferença entre a menor carga térmica e a maior foi de aproximadamente 3,5 vezes, calculadas usando Margules e UNIFAC. Ademais, disto inferese o alto impacto de um modelo termodinâmico que não se ajusta de modo adequado aos dados experimentais de equilíbrio sobre resultados simulados, partindo do pressuposto que os dados experimentais são fidedignos.

\section{CONCLUSÃO}

Neste trabalho foi mostrado o impacto que tem a escolha dos modelos termofísicos escolhidos em uma simulação usando um simulador de processos sobre os resultados obtidos. Nas etapas de projeto, análise, síntese e otimização de plantas inteiras, uma escolha equivocada dos modelos termofísicos pode levar a equipamentos super ou sub estimados. Se forem fixadas as condições de saída, como considerado neste trabalho, e também os parâmetros dos equipamentos, a discrepância será no gasto energético calculado, em 
processos envolvendo separação por colunas de destilação. Ademais, mostrou-se como os modelos termofísicos influenciam em quantificadores termodinâmicos da facilidade/dificuldade da separação, por exemplo, volatilidade relativa e taxa de transferência de massa, ao longo das colunas, ou seja, é possível com tais quantificadores determinar as regiões em uma coluna de destilação de maior incerteza e assim avaliar o impacto que isto tem no projeto destas e na influência sobre os valores calculados para as correntes de saída.

Tabela 5 - Influência do modelo termodinâmico para o cálculo do coeficiente de atividade do decantador na carga térmica dos trocadores de calor do processo

\begin{tabular}{|c|c|c|c|c|c|}
\hline \multirow{2}{*}{$\begin{array}{c}\text { Trocadores de } \\
\text { Calor }\end{array}$} & \multicolumn{5}{|c|}{ Cargas térmicas [MW] } \\
\cline { 2 - 6 } & NRTL & $\begin{array}{c}\text { UNIQUA } \\
\text { C }\end{array}$ & UNIFAC & $\begin{array}{c}\text { UNIFAC } \\
\text { modificado }\end{array}$ & Margules \\
\hline Da coluna C1 & 1,1115 & 1,1115 & 1,1115 & 1,1115 & 1,1115 \\
\hline $\begin{array}{c}\text { Antes do } \\
\text { decantador }\end{array}$ & $-2,0476$ & $-2,0941$ & $-2,3655$ & $-8,8310$ & $-1,9409$ \\
\hline Da coluna C2 & 0,2735 & 0,3198 & 0,5895 & 0,3291 & 0,1661 \\
\hline
\end{tabular}

\section{AGRADECIMENTOS}

Os autores agradecem à FAPEMIG (Processo PCE-00089-14) pelo apoio concedido.

\section{REFERÊNCIAS}

COCO - the CAPE-OPEN to CAPE-OPEN simulator, <http://www.cocosimulator.org/> acessado 24/02/2014.

DDBST GmbH, <http://www.ddbst.com/en/EED/VLE/VLE\%201-Butanol\%3BWater.php>, acessado dia 06/07/2013.

Góral, M.; Wisniewska-Goctowka, B.; Maczynski, A. Recommended Liquid-Liquid Equilibrium Data.Part 4.1-Alkanol-Water Systems. J. Phys. Chem. Ref. Data, 35 (2006), 1391-1414.

Hill, D.; Justice, F. C. Understand Thermodynamics to Improve Process Simulations. AIChE Journal, (2011), 20-25.

Luyben, W. L. Control of the Heterogeneous Azeotropic n-Butanol/Water Distillation System.Energy \& Fuel. 22(2008), 4249-4258.

Pinto, J. C. On the Costs of Parameter Uncertainties.The Effect of Parameter Uncertainties on the Optimization and Design of Experiments. Chem. Engng. Sci. 53(1998), 2029-2040.

Pinto, J. C. On the Costs of Parameter Uncertainties. 2. The Impact of EVOP Procedures on the Optimization and Design of Experiments. Can. J. Chem. Eng. 79 (2001), 412-421. 